\title{
ANÁLISE COMPARATIVA DE UM AQUECEDOR SOLAR CONVENCIONAL COM UM AQUECEDOR SOLAR DE BAIXO CUSTO
}

Isabela Santos Aragão - aragaobela@ eambjr.com.br Instituto Federal de Educação Ciência e Tecnologia da Bahia - IFBA Janiny Souza Silva - janiny.silva@ hotmail.com Instituto Federal de Educação Ciência e Tecnologia da Bahia - IFBA

Maira Caetano de Andrade - andrade.maira.2@gmail.com Instituto Federal de Educação Ciência e Tecnologia da Bahia - IFBA Maria Clara Gonçalves Santos - goncalvesantos.clara@ hotmail.com Instituto Federal de Educação Ciência e Tecnologia da Bahia - IFBA Roberta Rodrigues de Albuquerque - robertaalb14@gmail.com Instituto Federal de Educação Ciência e Tecnologia da Bahia - IFBA 


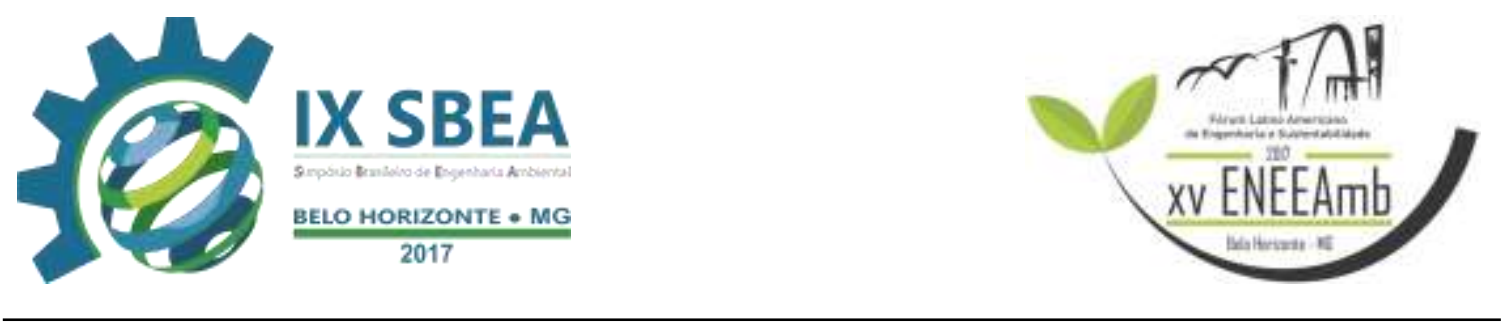

\section{RESUMO}

Aquecedores solares têm sido amplamente utilizados como fonte alternativa de energia. No entanto, os custos que envolve a instalação desses tipos de equipamentos são elevados. Neste contexto, insere-se pesquisas que visam testar a eficiência dos coletores solares de baixo custo. De tal forma, o presente trabalho estabelece um estudo comparativo da eficiência térmica de um sistema de aquecimento solar de água de baixo custo com um sistema convencional de aquecimento de água da marca Soletrol. Foi encontrado no aquecedor solar de baixo custo um coeficiente de perda de calor menor do que no convencional analisado. Isso também se refletiu na eficiência, tanto na eficiência média dos dias estudados quanto na média do dia.

Palavras-chave: aquecedor solar, baixo custo, eficiência térmica.

\section{INTRODUÇÃO/OBJETIVO}

Fontes de energia renováveis são aquelas em que os recursos naturais são usados e tem a capacidade de se reabilitar, são tidos como inesgotáveis (SOUZA, 2002). Consideradas como alternativas de energia limpa, as fontes renováveis de energia potencial são: energia eólica, de biomassa, maremotriz, geotérmica e solar. De acordo a Secretaria da Indústria, Comércio e Mineração da Bahia (2014), a busca por fontes alternativas de energia está entre as maiores preocupações do mundo atual. $\mathrm{O}$ alto custo da produção de energia, a demanda sempre crescente e os desafios do desenvolvimento com sustentabilidade geraram um grande interesse dos países em desenvolver formas e equipamentos para produção de eletricidade que contribuam para a redução da dependência do uso de combustíveis fósseis, e para busca por alternativas de energia limpa, como exemplos, a energia solar e a eólica.

$\mathrm{O}$ uso de aquecedores solares convencionais tem sido uma técnica adotada em todo o mundo como possibilidade de minimizar custos com a eletricidade e corroborar com a sustentabilidade, infelizmente a adoção de um sistema de aquecedor solar envolve um alto custo de instauração, tornando a alternativa economicamente inviável para populações de baixa renda. O Brasil apresenta grande potencial para utilização da energia solar, recebendo de 1500 a $2500 \mathrm{kWh} / \mathrm{m}^{2}$ de radiação solar incidente em todo o seu território (PEREIRA et al., 2006). Diante desse cenário, muitos estudos vêm sendo 


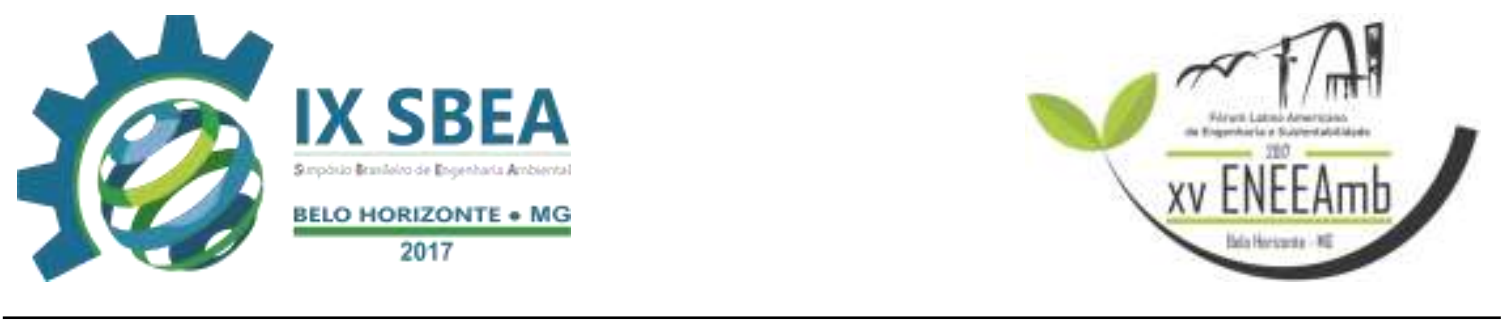

realizados para testar a eficiência dos coletores solares de baixo custo. O presente trabalho tem como objetivo um estudo comparativo da eficiência térmica de um sistema de aquecimento solar de água de baixo custo com um sistema convencional de aquecimento de água da marca Soletrol. Visando ser implantado e utilizado para fins residenciais na cidade de Vitória da Conquista/BA o sistema de aquecimento com melhor desempenho e custo benefício.

\section{METODOLOGIA}

Nos dois sistemas de aquecimento solar analisados foi adotado o princípio de termossifão ou circulação forçada, ou seja, o movimento da água se dá através da diferença de densidade entre as águas do reservatório e do coletor. A água do coletor sofre a incidência direta do sol ficando mais quente e, portanto, menos densa que a água no reservatório. Esta, por sua vez, por ser mais densa e, portanto, mais pesada, empurra a água quente do coletor, gerando a circulação.

O sistema de aquecedor solar de baixo custo teve o seu coletor solar construído de garrafas PET e embalagens Tetra Park, acondicionando tubos de PVC por onde ocorre a circulação da água. O sistema também é composto pelo reservatório térmico que consiste em uma bombona plástica revestida de material isolante de baixo custo. $\mathrm{O}$ coletor solar do sistema de aquecedor solar convencional empregado no estudo foi da marca Soletrol modelo Max Alumínio. A estação meteorológica utilizada, Davis Vantage Pro 2, forneceu os dados relativos à temperatura ambiente e à radiação solar. Para se determinar a eficiência dos dois coletores solar e o coeficiente global de perdas do sistema de aquecimento solar foi utilizado a metodologia de Guerra e Varella. Para isto, foram coletados dados de dez em dez minutos no período entre 8:00 e 15:00 horas dos dias 3 a 16 de fevereiro de 2016. Para a realização da medição da temperatura da água na entrada e saída da placa coletora foram utilizados sensores digitais do tipo DS18B20.

\section{RESULTADOS E DISCUSSÃO}

O gráfico apresentado na Figura 1 mostra as oscilações dos rendimentos calculados do coletor solar do sistema de aquecedor solar de baixo custo bem como 


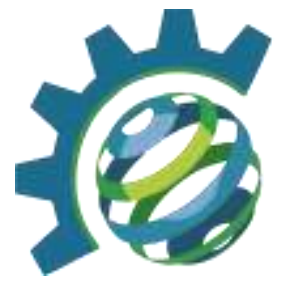

destaca o rendimento médio no período estudado. Já o gráfico mostrado na Figura 2 apresenta as oscilações do rendimento do coletor solar do sistema de aquecedor solar convencional.

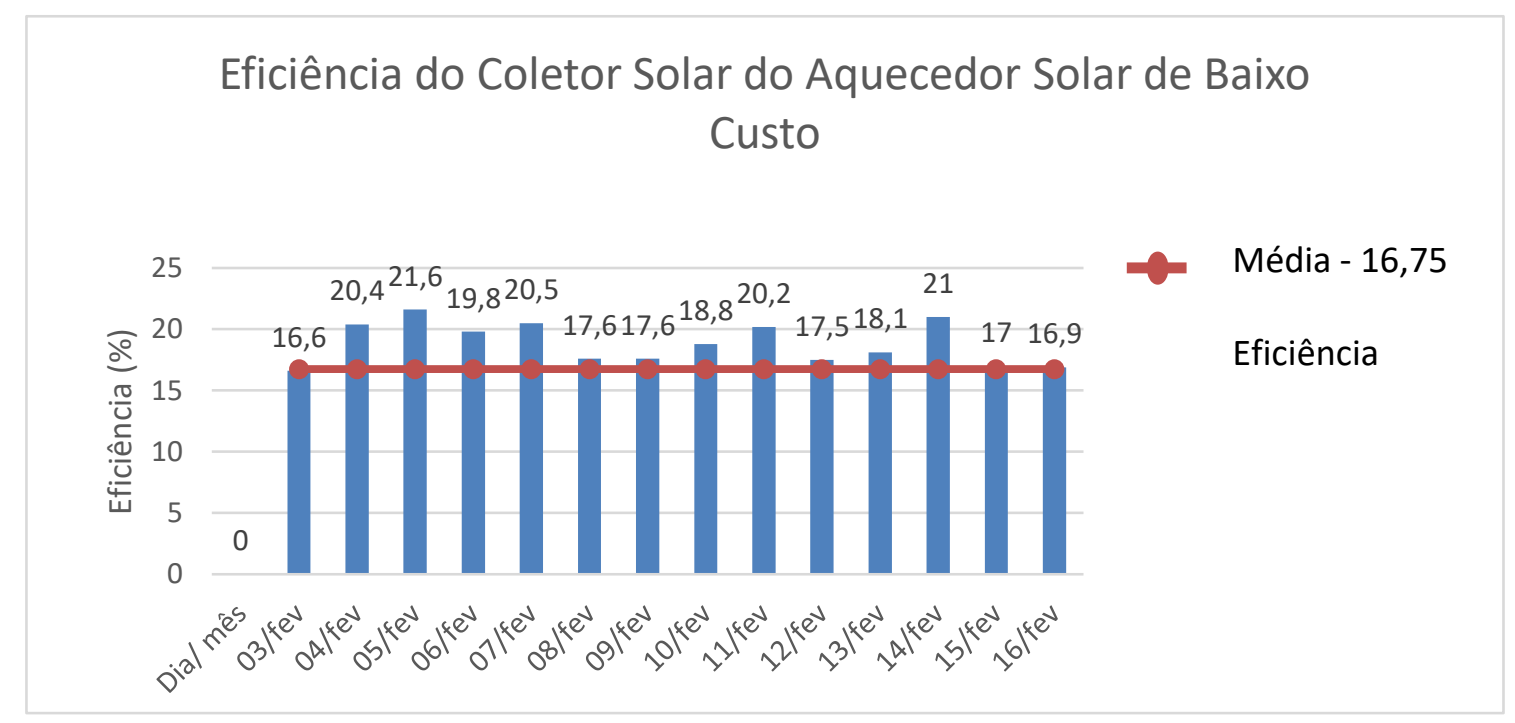

Figura 1- Eficiência do Coletor Solar do Aquecedor Solar de Baixo Custo.

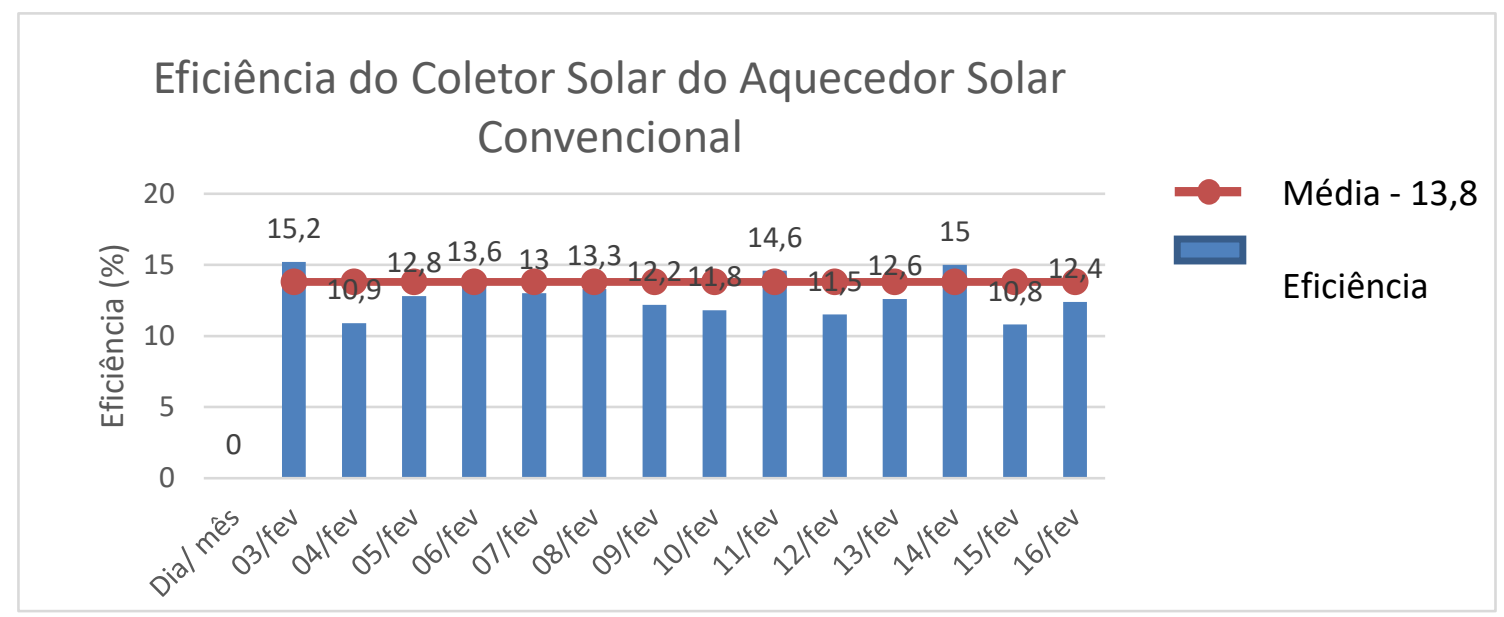

Figura 2- Eficiência do Coletor Solar do Aquecedor Solar Convencional.

Na Figura 1 é possível perceber que o valor máximo de eficiência foi atingido no dia 05 de fevereiro, $21.6 \%$. Isso é justificado pelo fato de ter alcançado uma diferença de $12,6{ }^{\circ} \mathrm{C}$ entre as temperaturas da água na entrada e saída da placa coletora, mesmo 


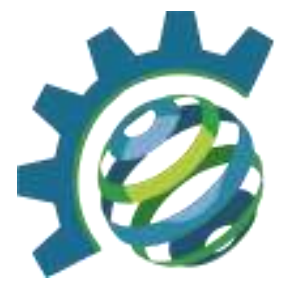

com a radiação solar com um valor médio de 483,74 W/m², inferior aos valores observados no período monitorado. O valor máximo de temperatura medido na saída da placa foi de $65^{\circ} \mathrm{C}$ e o mínimo de $49,3^{\circ} \mathrm{C}$. Já na Figura 2, a partir da análise do gráfico, percebe-se que o dia em que verificou a maior eficiência, foi no dia 03 de fevereiro, $15,2 \%$, com a média de incidência de radiação solar $586,53 \mathrm{~W} / \mathrm{m}^{2}$ com o valor máximo de temperatura medido na saída da placa de $60,4{ }^{\circ} \mathrm{C}$ e o mínimo de $43,3{ }^{\circ} \mathrm{C}$.

As Figuras 3 e 4 mostram os gráficos do coeficiente global de perdas dos coletores solar calculados (baixo custo e convencional). Tal coeficiente está relacionado com a energia térmica perdida para o ambiente $(\mathrm{kW})$ e com a diferença de temperatura entre a placa $(\mathrm{K})$ e o ambiente, além da área da superfície da placa $\left(\mathrm{m}^{2}\right)$.

\section{Coeficiente Global de Perda do Aquecedor Solar de Baixo}

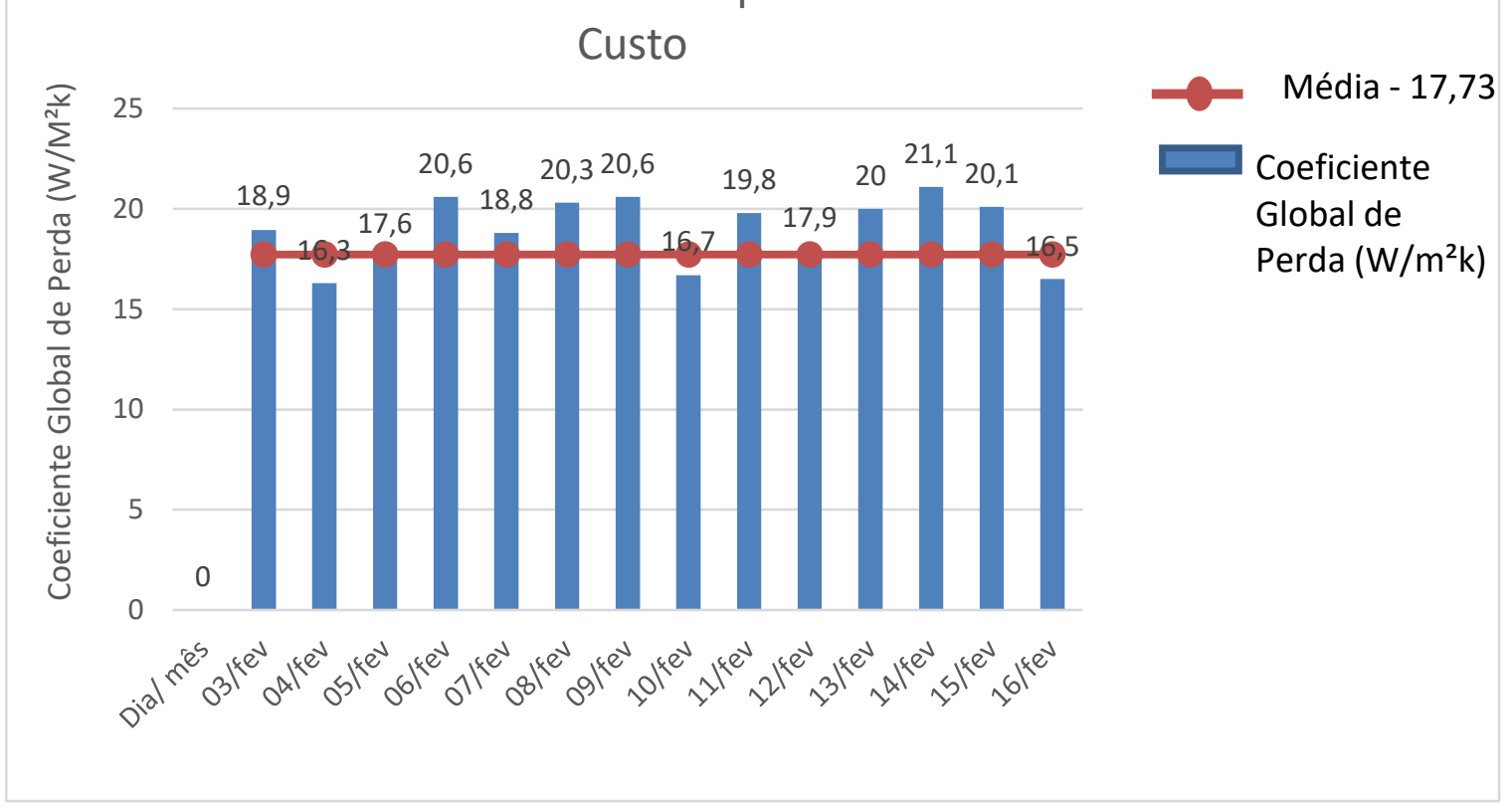

Figura 3 - Coeficiente Global de Perda do Aquecedor Solar de Baixo Custo.

Com média do coeficiente de perda de $17,73 \mathrm{~W} / \mathrm{m}^{2} \mathrm{k}$ no Aquecedor Solar de Baixo custo não foi muito discrepante do coeficiente de perda dos coletores convencionais, que é uma média de $12 \mathrm{~W} / \mathrm{m}^{2} \mathrm{k}$. Esperava-se este resultado, visto que coletores de baixo custo não apresentam isolamento adequado que os torne irrelevantes às trocas convectivas entre o coletor em estudo e o ambiente. 

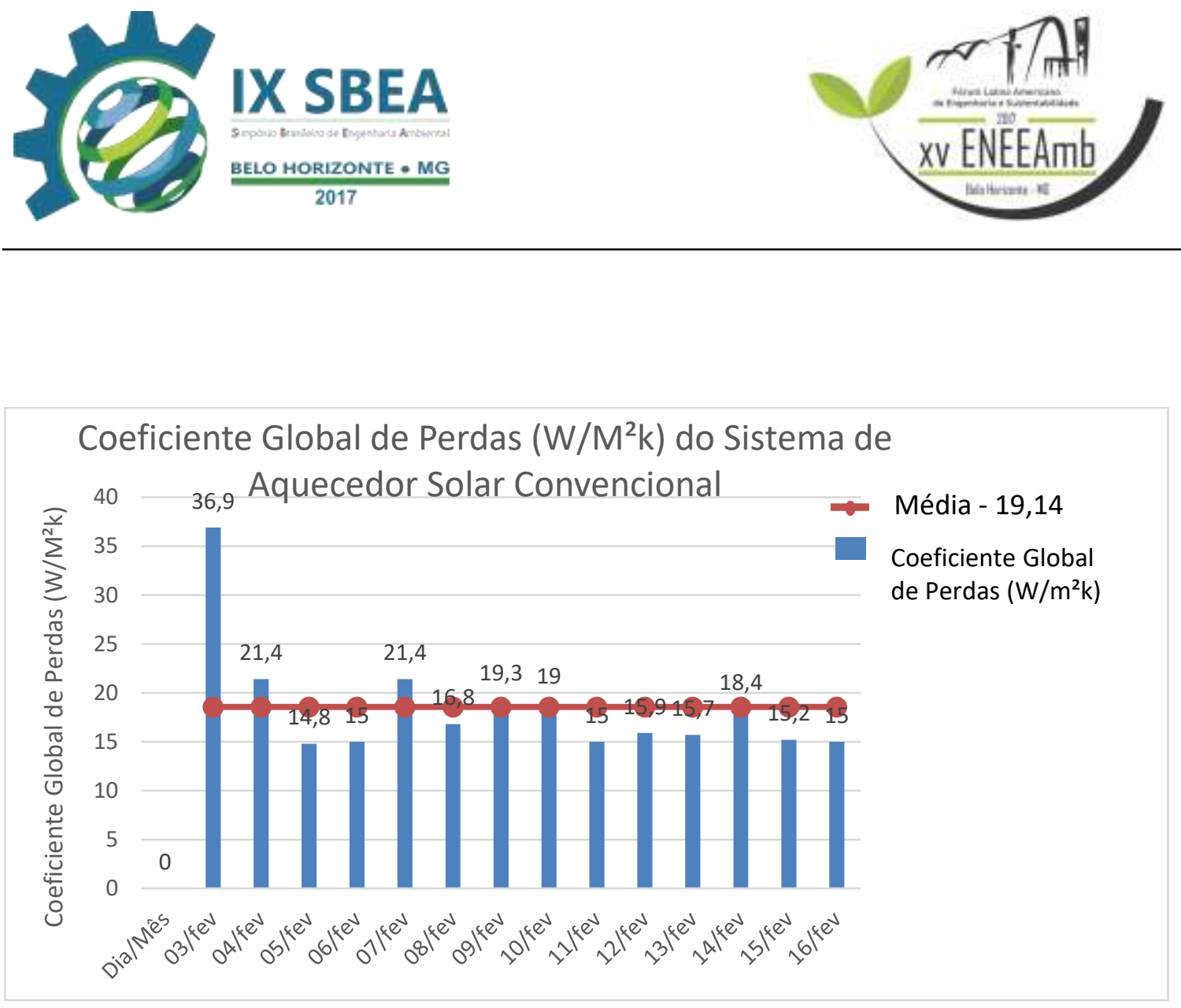

Figura 4 - Coeficiente Global de Perdas do Sistema de Aquecedor Solar Convencional.

Com valor médio de $19,14 \mathrm{~W} / \mathrm{m}^{2} \mathrm{~K}$ o coeficiente de perda do aquecedor convencional foi relativamente alto, visto que a média destes, como já citado a cima é de $12 \mathrm{~W} / \mathrm{m}^{2} \mathrm{k}$. Foi encontrado no aquecedor solar de baixo custo um coeficiente de perda de calor menor do que no convencional também analisado, pode-se concluir com isso que o material de isolamento térmico da Placa de baixo custo tem uma capacidade de reter calor maior do que a convencional. Isso também se reflete na eficiência, tanto na eficiência média dos dias estudados, tanto na média do dia, em que um valor percentual maior foi encontrado no coletor do aquecedor solar de baixo custo.

\section{CONCLUSÕES/RECOMENDAÇÕES}

A eficiência térmica (ou rendimento térmico) do coletor em estudo variou entre $16,6 \%$ e $21,6 \%$, que não é um valor muito discrepante de outros coletores de baixo custo, embora os coletores solares convencionais apresentem normalmente eficiência térmica superior a 55\%. Porém, o coletor solar foi capaz de atingir níveis de temperatura entre $49,3{ }^{\circ} \mathrm{C}$ e $65^{\circ} \mathrm{C}$ durante o período estudado, sendo superior à média considerada ideal para banho $\left(36^{\circ} \mathrm{C}\right)$. Mostrando, assim, um bom desempenho. 


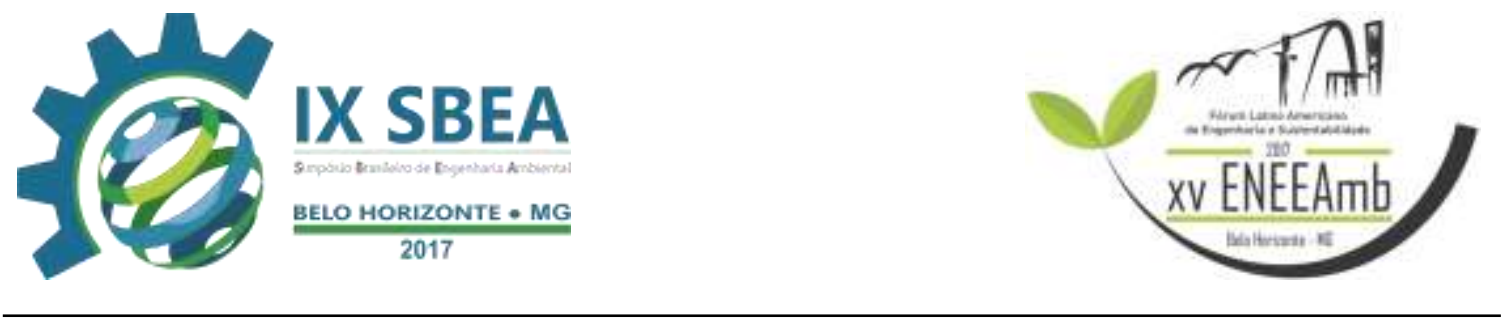

Verificou-se também que as perdas térmicas não são muito superiores em relação às dos coletores convencionais, sendo até menores que as perdas de outros modelos de sistemas alternativos e de baixo custo estudados por demais pesquisadores.

\section{REFERÊNCIAS BIBLIOGRÁFICAS}

BAHIA (Estado). Secretária da Indústria, Comércio e Mineração. Diretoria de Desenvolvimento e Relações Internacionais e Superintendência de Desenvolvimento Econômico. Energia Eólica. Bahia, 3 p.

GUERRA, M. I. S. e VARELLA, F. O. M. Análise do Desempenho Térmico de um Sistema Solar de Baixo Custo na Cidade de Mossoró (RN). HOLOS, Ano 30, Vol. 4, 2014.

PEREIRA, E. B; MARTINS, F. R; ABREU, S. L. e RÜTHER, R. Atlas Brasileiro de Energia Solar. $1^{\mathrm{a}}$ ed. São José dos Campos. 2006.

SOUZA, L. G. M; GOMES, U. U. Viabilidade Térmica, Econômica e de Materiais de Utilização de Tubos de PVC como Elementos Absorvedores em Coletores de um Sistema de aquecimento de Água por Energia Solar. Tese do Programa de Doutorado em Ciência e Engenharia de Materiais, UFRN, 2002. 